\title{
Improved Efficient RFID Tag Estimation Scheme
}

\author{
Mian Hammad Nazir 1 Shahid Mehmood 2 Nathirulla Sheriff 3 Ahsan Adeel4 \\ Dept. of Electrical Engineering, COMSATS Institute of Information Technology. Islamabad. Pakistan 1,2,4 \\ Dept. of Electronics Engineering, Sri Krishna College of Technology. India 3
}

\begin{abstract}
DFSA (Dynamic Frame Slotted Aloha) based anti-collision algorithms resolve the collision among the RFID (Radio frequency-ID) tags by adjusting the frame size for the incoming frame according to the number of un-identified tags. The system efficiency directly depends upon the estimated number of tags that contribute towards the frame size adjustment. In this paper, we propose an improved scheme for DFSA which accurately estimates the tags that have to participate in the incoming frame. The scheme also adjusts the incoming frame size according to the estimated tags and keeps the system efficiency very close to the optimal. The simulation results show that for the proposed scheme the tag estimation time and the estimation error rate is far less compared to conventional methods and system efficiency is close to optimal.
\end{abstract}

\section{Keywords}

Passive UHF RFID, Tag estimation scheme, EPC global class 1 Gen 2, maximum system efficiency

\section{INTRODUCTION}

RFID based systems can be broadly categorized in to two broad fields mainly near field RFID systems and far field RFID systems. Compare to near field RFID systems which make use of magnetic induction, the range of far field RFID systems is more i.e., many tags can be identified within readers range. Far field RFID systems make use of electro-magnetic waves [1].

As the number of tags increases within the readers range an efficient tag to tag anti-collision algorithm is needed. These tag to tag anti-collision algorithms can broadly be categorized in to Tree based and ALOHA based. Among ALOHA based protocols there are ALOHA, Slotted ALOHA and framed-slotted ALOHA based protocols. Frame slotted ALOHA is the most popular among all these. Tag estimation techniques are important, as for optimal system efficiency number of tags should be equal to the frame size i.e. $\mathrm{n}=\mathrm{N}$. The frame size need to be adjusted using some efficient anti-collision algorithm. An efficient anti-collision algorithm adjusts the frame size according to the estimated number of tags with system efficiency very much close to optimal. But in order to achieve maximum system efficiency the estimated number of tags must also have very less error estimation rate and estimation results should be close or equal to optimal. Our proposed tag estimation is designed in order to attain maximum tag estimation efficiency.

This paper is organized as follows. In Section 2, we explain major conventional tag estimation schemes. In Section 3, we propose our idea of efficient tag estimation. In Section 4 performance analysis and comparison through simulations is presented and we conclude in Section 5.

\section{CONVETIONAL TAG ESTIMATION SCHEMES}

A Framed-slotted ALOHA protocol uses slotted-ALOHA which is made by applying discrete time division and further includes grouping of slots into frames with each frame having $\mathrm{N}$ slots. Dynamic frame slotted ALOHA (DFSA) [6] is one of the most commonly used schemes for various RFID systems. Each tag randomly selects a slot to transmit its data so the factors that greatly add to the system efficiency are frame size and number of tags (tag population).The size of the frame is adjusted according to tag population. The reader don't have any information about the tag population so it decides the frame size on the basis of collision ratio and idle slot ratio. The schemes that estimate the number of tags on the basis of collision and idle slot ratio and then adjust the frame size according to these are discussed below.

\subsection{Schoute Backlog Estimation method}

Schoute [2] proposed a DFSA method using poisson distribution. Schoute's backlog estimation technique multiplies the number of collision slots in a frame by the expected value of 2.39 tags per collision slot to estimate the number of unidentified tags.

$$
B_{t}=2.39 c
$$

where "c" represents the number of collision slots in a frame .This method shows good results when the number of tags is close to number of slots. However the performance becomes poorer when the difference between tags and slots increases.

\subsection{Lower Bound backlog estimation}

\section{method}

Lower bound backlog [2] tag estimation method states that the estimation function is obtained with the assumption that a collision involves at least two different tags. So backlog after the recent frame is given as

\subsection{Vogt method}

$$
B_{t}=2 c
$$

Vogt proposed to use the vector distance between the read result and the expected value vector to determine the tag quantity. As for every collision slot two tags are involved so lower bond for tag quantity is given as

$$
n=C_{1}+2 C_{\geq 2}
$$

Lower bond backlog estimation has been discussed in the previous section. In $C_{1}$ is the number of slots with one tag each (successful slots) and $C_{\geq 2}$ is the number of slots with multiple tags (collision slots). Vogt used the Markov process to decide the frame size in each round cycle and modeled a complete read process[3][4].

\subsection{Chebyshev's Inequaltiy estimation method}

According Chebyshev's inequality, the outcome of a random experiment involving a random variable $\mathrm{X}$ is most likely somewhere near the expected value of $\mathrm{X}$. Thus estimation function measures the difference between the real results and the expected values to estimate the number of tags for which difference becomes minimal [4]. 
$\varepsilon_{\mathrm{vd}\left(\mathrm{N}, \mathrm{c}_{0}, \mathrm{c}_{1}, \mathrm{c}_{\mathrm{k}}\right)}=\min \left|\left(\begin{array}{c}a_{o}^{N, n} \\ a_{1}^{N, n} \\ \mathrm{~N}_{\geq 2}, \mathrm{n} \\ \mathrm{N}_{\geq 2}\end{array}\right)-\left(\begin{array}{c}c_{o} \\ \mathrm{c}_{1} \\ \mathrm{c}_{\mathrm{k}}\end{array}\right)\right|$

The number of tags is estimated using both the number of slots $\mathrm{N}$ used in the read cycle and the results of the previous read cycle as a triple of numbers $\left(c_{0}, c_{1}, c_{k}\right)$ that quantify respectively the empty slots, slots filled with one tag, and slots with collision as Equation(1). In Eq.(4). ( $\mathrm{a}_{\mathrm{o}}^{\mathrm{N}, \mathrm{n}}, \mathrm{a}_{1}^{\mathrm{N}, \mathrm{n}}, \mathrm{a}_{\geq 2}^{\mathrm{N}, \mathrm{n}}$ ) respectively denote the empty slots, slots filled with one tag, and slots with collision where the number of slots is $\mathrm{N}$ and the number of tags is $\mathrm{n}$. Given $\mathrm{N}$ slots and $\mathrm{n}$ tags, the number $0,1, \mathrm{r}$ of tags in one slot is binomially distributed, and the expectation value for them is given by the follow Eq.(5)[5].

$$
\mathrm{a}_{\mathrm{r}}^{\mathrm{n}, \mathrm{N}}=(X=r)=\mathrm{N} \cdot\left(\begin{array}{l}
n \\
r
\end{array}\right)\left(\frac{1}{N}\right)^{r}\left(1-\frac{1}{N}\right)^{n-r}
$$

\subsection{Zhen estimation method}

This method [9] estimates the tags as follows. The probability distribution function for $\mathrm{n}$ tags as they choose a slot is given as,

$$
p_{n}^{0}(i)=\left\{\begin{array}{ll}
0 & \text { if } \mathrm{n}=0,1 \\
\frac{\mathrm{p}_{\mathrm{n}}(\mathrm{i})}{1-\mathrm{p}_{0}(\mathrm{i})-\mathrm{p}_{1}(\mathrm{i})} & \text { if } \mathrm{n} \geq 2
\end{array}\right\}
$$

From Eq (6), number of tags that collided inside a single slot is,

$\lim _{N \rightarrow \alpha} \sum_{k=2}^{N}\left(\mathrm{k}_{\mathrm{k}}^{\mathrm{p}^{0}}\right)(\mathrm{i})=2.39=\mathrm{k}$

This relationship specifies that for a collided slot 2.39 tags respond. So this method states that the total number of tags that are estimated are

$$
\mathrm{c} 1+2.39 \mathrm{cn} \text {. }
$$

\subsection{Chen and Lin methods}

Chen and Lin method [5] basically comprises of two main parts First one is to calculate estimate the number of tags according to the Triple parameter (E,S,C). After the first read cycle is completed the reader now can estimate the number of collision, idle and successful slots as,

$$
\text { Frame size }(N)=C+S+E
$$

The other part is to estimate the optimal frame size in order to attain maximum system efficiency. A well know Feller equation from which we can get the probabilty of finding exactly $\mathrm{N}$ slots against $n$ tags. This is same as situation of $m$ boxes that need to fit $\mathrm{k}$ balls in them. This equation is given as.

$$
P(k, m)=\frac{(-1)^{m} N ! n !}{m ! N^{n}} \cdot \sum_{j=m}^{\min \left(N,\left[\frac{n}{k}\right]\right)}(-1)^{\mathrm{j}} \cdot \frac{(\mathrm{N}-\mathrm{j})^{\mathrm{n}-\mathrm{jk}}}{(\mathrm{j}-\mathrm{m}) !(\mathrm{N}-\mathrm{j}) !(\mathrm{n}-\mathrm{jk}) !(\mathrm{k} !)^{\mathrm{j}}}
$$

Chen and Lin used two methods to estimate the number of tags. First one was to estimate tags on the basis of empty slots after one read cycle.From Eq.(10) we can derive the probabilty of finding empty slots as,

$$
P(e)=\frac{(-1)^{e} N ! n !}{e ! N^{n}} \cdot \sum_{j=e}^{\mathrm{N}}(-1)^{\mathrm{j}} \cdot \frac{(\mathrm{N}-\mathrm{j})^{\mathrm{n}}}{(\mathrm{j}-\mathrm{e}) !(\mathrm{N}-\mathrm{j}) !(\mathrm{n}) !}
$$

Chen and Lin calculated number of tags ' $n$ ' under a condition for the maximum probailty for the given value of $\mathrm{N}$ and empty slots e.

The second method was based on the average value of empty slots e and succesfull slots s. Using binomial distribution function which gives number of tags for a given slot with the probability of $1 / \mathrm{N}$. So probabilty of finding $r$ tags in a slot is given as.

$$
P_{r_{n, N}}(X=r)=\left(\begin{array}{l}
n \\
\mathrm{r}
\end{array}\right)\left(\frac{1}{\mathrm{~N}}\right)^{\mathrm{r}} \cdot\left(1-\frac{1}{\mathrm{~N}}\right)^{\mathrm{n}-r}
$$

The expected number of empty slots $a_{0}$ is calculated from Eq. (12)

$$
a_{0}=N \cdot P_{r_{n, N}}(X=0)=N \cdot\left(1-\frac{1}{\mathrm{~N}}\right)^{\mathrm{n}}
$$

And the expected number of succesful slots $a_{1}$ is given as,

$$
a_{1}=N \cdot P_{r_{n, N}}(X=1)=n \cdot\left(1-\frac{1}{\mathrm{~N}}\right)^{\mathrm{n}-1}
$$

Solving Eq.(13) and Eq.(14) they obtained the follwoing equation

$$
n=(N-1) \cdot\left(\frac{a_{1}}{a_{0}}\right)
$$

There fore the number of unread tags were obtained by subtracting the succesful slots from the number of tags found above in Eq (16)

$$
\text { Total unread Tags }=\left(N-a_{0}-1\right) \cdot\left(\frac{a_{1}}{a_{0}}\right)
$$

Chen Lin method 1 shows better performance compared to Vogt even with higher number of tags while Chen Lin method 2 performance is considerable poor compared with Vogt and method 1 at higher number of tags.

\section{THE PROPOSED ESTIMATION SCHEME}

Our proposed Tag estimation scheme estimates the total number of tags that will participate in the next coming frame and then depending upon this estimation the next frame size is adjusted. The more the accurately the tags are estimated the more the efficiently the frame size will be adjusted for upcoming frame. Thus our scheme shows optimal results compared with conventional methods [2]-[9] by efficiently estimating the tags and adjusting the frame size keeping system efficiency close to optimal.

Let's assume that system efficiency is given as,

$$
\beta=\frac{\text { number of successful slots }}{\text { frame size }}=\frac{\mathrm{n}_{\mathrm{s}}}{\mathrm{N}}
$$

Eq.(18) shows that the frame size $\mathrm{N}$ is equal to the product of factor $\alpha$ and the number of tags $n$.So for this we have condition for Number of tags and frame size as,

$$
N=\alpha \cdot n
$$

Where $\alpha$ denotes the ratio of frame size " $\mathrm{N}$ " to the number of tags ' $n$ '. As it is clear that,

$$
\begin{aligned}
& n=n_{e}+n_{s}+n_{c} \\
& \alpha=\frac{N}{n_{e}+n_{s}+n_{c}}
\end{aligned}
$$

From Eq.(17) and (18) 


$$
\beta=\frac{n_{s}}{\alpha \cdot n}
$$

Putting Eq.(19) in Eq.(21)

$$
\beta=\frac{n_{s}}{\alpha \cdot\left(n_{e}+n_{s}+n_{c}\right)}
$$

Solving Eq.(22)

$$
\begin{aligned}
& \frac{n_{s}}{\alpha \cdot \beta}-n_{s}=n_{e}+n_{c} \\
& n_{s}-\alpha \beta \cdot n_{s}=\alpha \beta\left(n_{e}+n_{c}\right) \\
& \frac{n_{s} \cdot(1-\alpha \beta)}{\alpha \beta}=n_{e}+n_{c}
\end{aligned}
$$

The relationship graph between $\alpha$ and $\beta$ is shown in fig.1 Adding and subtracting $n_{s}$ on L.H.S of Eq.(25),

$$
\begin{aligned}
& n-n_{s}=\frac{n_{s} \cdot\left(1-\left(\frac{n_{s}}{N}\right)\left(\frac{N}{n}\right)\right)}{\frac{n_{S}}{n}} \\
& n-n_{s}=n \cdot\left(1-\frac{n_{s}}{n}\right)
\end{aligned}
$$

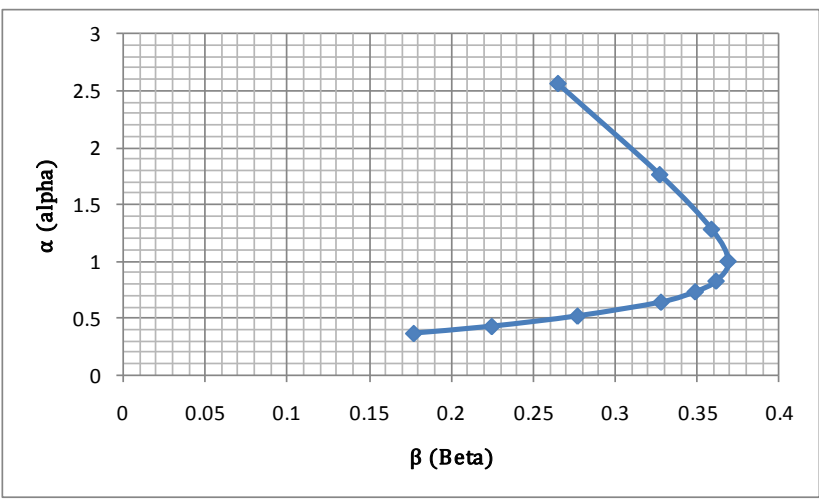

Fig.1 Relationship between $\alpha$ and $\beta$

The number of tags that remain behind (for determination of next frame size) will be equal to the total number of tags minus the tags that are successfully identified so , $n-n_{s}=$ unidentified tags.

In the previous section 3 from Eq. (11) the expected number of successful slots due to successfully identified tags is given as,

$$
\begin{aligned}
& n-n_{s}=n \cdot\left(1-\left(\frac{n \cdot\left(1-\frac{1}{N}\right)^{n-1}}{n}\right)\right. \\
& n-n_{s}=n \cdot\left(1-\left(1-\frac{1}{N}\right)^{n-1}\right) \\
& n-n_{s}=n \cdot\left(1-\left(\frac{1}{e}\right)^{\frac{n-1}{N}}\right)
\end{aligned}
$$

Eq.(31) shows the estimated number of tags that need to be identified in the coming frame. Next frame size is decided on the basis of these unidentified tags (i.e. Back $\log$ )

$$
n-n_{s}=n \cdot\left(1-e^{-\frac{N}{n-1}}\right)
$$

Thus if,
$\left(1-e^{-\frac{N}{n-1}}\right)=\gamma$

Eq.(32) can be written in terms of $\alpha$ as shown in Eq.(33).

$\left(1-e^{-\left(\left(\frac{1}{\alpha}\right)-N\right)}\right)=\gamma$

$n-n_{s}=n \cdot \gamma$

So the accuracy of the estimated tags will depend upon the exponential function $\gamma$.

Fig 2. Shows the relationship between $\alpha$ and $\gamma$, from the graph it can be clearly seen that $\alpha$ and $\gamma$ are inversely related to each other. As $\alpha$ (ratio of $\mathrm{N}$ to $\mathrm{n}$ ) increases which means that as $\mathrm{n}$ approaches close to $\mathrm{N}, \gamma$ decreases and vice versa.

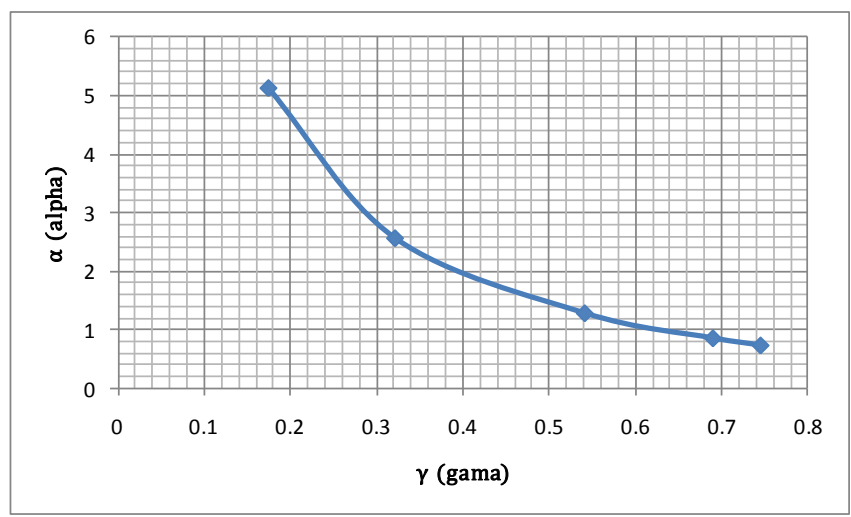

Fig.2.Relationship between $\alpha$ and $\gamma$

The frame size can be adjusted according to the estimated number of tags by using the pseudo-code in fig 3 . This pseudo-code uses dynamic grouping approach and adjusts the frame size "N" depending upon two conditions i.e. if estimated number of tags $>$ frame size and vice versa.

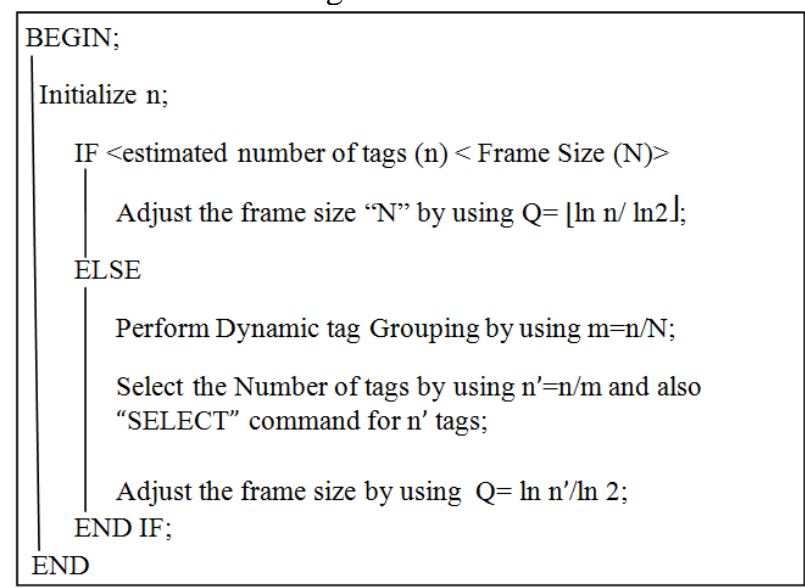

Fig.3 Pseudo-code for adjusting frame size "N"

\section{PERFORMANCE ANALYSIS OF PROPOSED SCHEME}

The graph in fig. 4 shows the estimation error rate (ERR).ERR is calculated by taking the mean difference of estimated number of tags and the real number of tags. 


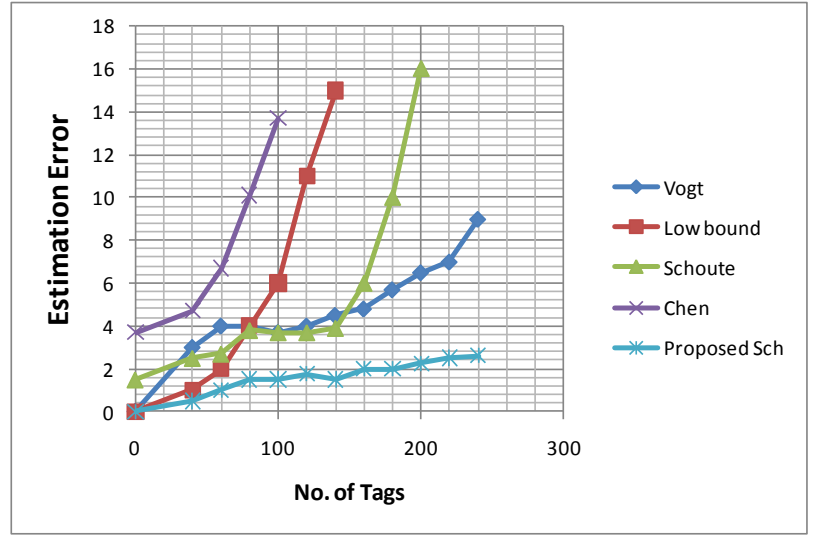

Fig.4. ERR comparison with conventional schemes.

In the simulation study the frame size was set equal to 128 and the number of tags were varied.

From the simulation study we can see that Schuotes and Vogt algorithms have nearly similar behavior in the beginning but as the number of tags increase Vogt algorithm shows comparatively good results. From the graph we can see that our proposed algorithm method shows good simulation results compared to Vogt and Schoute algorithms. The estimation error rate is less compared to conventional estimation algorithms.

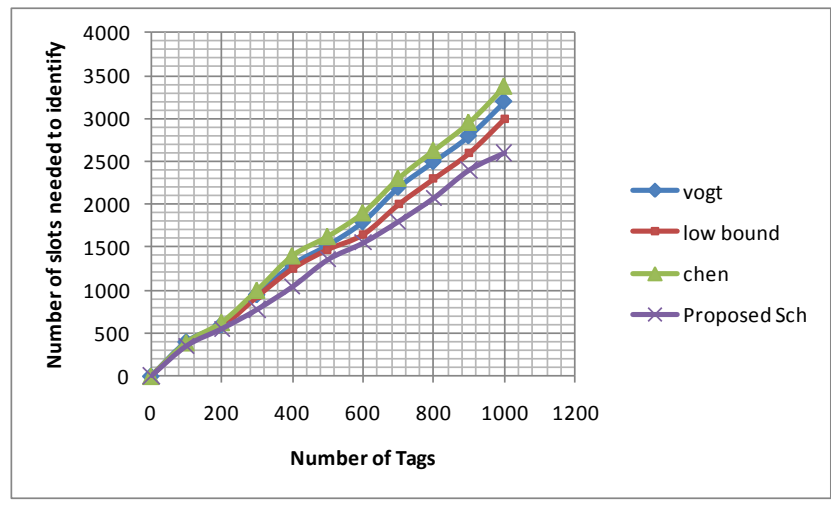

Fig.5. Number of slots needed to identify the tags

Fig. 5 shows the number of slots needed to identify the estimated number of tags. Initially when the number of tags are less than 300 , nearly all the schemes show the similar behavior but simulation results show better performance for our proposed scheme as the number of tags keep on increasing which shows that our proposed scheme is much efficient in case of slot utilization.

\section{CONCLUSION}

The paper presents an improved efficient tag estimation scheme which accurately estimates the unidentified tags and in turn adjusts the frame size of the next frame. The proposed scheme estimates the unidentified tags by calculating an exponential function $\gamma$ and multiplying it with the total number of tags, thus accuracy of tag estimation depends upon $\gamma$, which is calculated by analyzing successful and collided slots. The simulation results show that our proposed scheme reduces the estimation error rate and tag estimation time and improves the accuracy of tag estimation and takes less time slots to identify all the tags.

\section{REFERENCES}

[1] K.Finkenzeller and R. Waddington, RFID Handbook: Radio-Frequency Identification Fundamentals and Applications, John Wiley \& Sons, January2000. EPCglobal, EPC. Radio-frequency identity protocols class-1 generation2 UHF rfid protocol for communications at $860 \mathrm{MHz}-$ $960 \mathrm{MHz}$

version technology.Version1.2.0.2008.http:|lwww.epcglobalinc.org| standards।

[2] Dynamic Framed-Slot ALOHA Anti-Collision using Precise Tag Estimation Scheme Prapassara Pupunwiwat Bela Stantic ,1983

[3] Harald vogt ,"Efficient object identification with passive RFID tags". In international conference on pervasive computing, LNCS.Springer-Verlag,2002

[4] Harold vogt, "Multiple object identification with passive RFID tags," 2002 IEEE International Conference on Systems, Man and Cybernetics, Oct. 2002

[5] W-T chen and G.H Lin " An efficient anti-collision method for tag identification in RFID"IEICE TRANS.COMMUN.VOL.E89-B.NO.12- 2006

[6] C. Floerkemeier, "Bayesian transmission strategy for framed ALOHA based RFID protocols ," 2007 IEEE International Conference on RFID,March 2007

[7] Su-Ryun Lee, Sung-Don Joo, and Chae-Woo Lee, "An Enhanced Dynamic Framed SlottedALOHA Algorithm for RFID Tag Identification," The Proceeding of the 2nd Annual InternationalConference on Mobile and Ubiquitous Systems, 2005.

[8] Xu Huang, "An Improved ALOHA Algorithm for RFID Tag Identification," Lecture Notes in Computer Science.Springer Berlin/ Heideberg, Volume 4253/2006

[9] D. K. Klair, K.-W. Chin, and R. Raad, "On the accuracy of RFID tag estimation functions," International Symposium on Communication and Information Technologies, ISICT'07, pp. 1401-1406, 17-19 October 2007. 